\title{
Movement-induced modulation of orientation-specific color aftereffects
}

\author{
H. H. MIKAELIAN \\ University of Georgia, Athens, Georgia $\$ 0602$
}

\begin{abstract}
The intensity of the McCollough effect is modified when, following exposure to the inducing chromatic stimuli, the achromatic test gratings are seen oscillating orthogonally to their orientations. Green aftereffect seen on stationary test gratings is enhanced by oscillations, while pink aftereffect present on the stationary gratings fades upon oscillation of the test stimulus. These opponent changes are tentatively accounted for in terms of an interaction between Fechner-Benham type induced color and processes that mediate the orientation-specific chromatic aftereffects.
\end{abstract}

Orientation-specific chromatic aftereffects, also known as McCollough effects (McCollough, 1965), are typically generated by viewing stripes of one color in one orientation, such as green vertical grating, and those of another color in another orientation, such as red horizontal grating, in alternating sequence for several minutes. Achromatic stripes of similar spatial frequency (test stimuli) subsequently viewed appear tinted with the complementary of the original (adapting) colors when their orientation matches that of the adapting stripes, in this case, pink when oriented vertically and green when oriented horizontally. Since McCollough's initial report, a number of investigators have explored the phenomenon using features other than oriented gratings, such as movement (Favreau, Emerson, \& Corballis, 1972; Hepler, 1968; Mayhew \& Anstis, 1972). frequency of gratings (Leppman, 1973), angular and curvilinear gratings (Riggs, 1973; White \& Riggs, 1974), etc.

The present study concerns variations in the saturation of the chromatic aftereffect that occur when the achromatic test gratings, viewed to elicit these aftereffects following adaptation, are presented in motion. In a pilot study designed to determine whether or not better detectability of the achromatic test grating would enhance the saturation of the chromatic aftereffects, low-frequency gratings in oscillatory motion were used to test for the aftereffect, following induction by the usual stationary inducing chromatic stimuli. That procedure was based on the reasoning that temporal modulation of a lowfrequency grating enhances its detectability (Robson, 1966). The results showed that the apparent saturation of the McCollough effect did indeed change when the achromatic test stimuli were seen in motion. These changes, however, were found to depend upon the color of the aftereffect. The green aftereffect, which appeared on vertical achromatic grating, became more intense upon lateral oscillation of the test stimulus, while the pink aftereffect, which appeared on the horizontal grating, faded when that stimulus was made to oscillate vertically. To pursue systematically what appeared to be opponent changes in chromatic saturation of the orientation-specific aftereffects by stimulus motion, and to determine whether such changes could be obtained at orientations other than the vertical and horizontal, the following experiment was conducted.

\section{METHOD}

Subjects

Twenty subjects with normal color vision, as tested by the American Optical H-R-R pseudoisochromatic charts, and with no previous experience with the McCollough effect were recruited.

\section{Apparatus and Procedure}

The adapting stimuli consisted of square-wave gratings, each bar having a spatial frequency of $3 \mathrm{cpd}$. The size of the entire field covered an area of $12^{\circ} \times 10^{\circ}$. The gratings were projected $(300-\mathrm{W}$ Sylvania CLX/CMB light source) through green and red filters (Wratten 25 and 58 ) on an aluminized screen. The luminances of the red, green, and black bars were 16,14 , and $2 \mathrm{~mL}$, respectively. The green and red stripes were viewed, alternately, for 15-sec intervals for a total viewing time of $5 \mathrm{~min}$. The achromatic test pattern, viewed under diffused flourescent lights, consisted of photographically reproduced high-contrast square-wave gratings (same frequency as adapting stimuli) of concentric rectangular patterns; it thus contained stripes in orthogonal orientations which. following adaptation, appeared tinted green and pink when oriented appropriately. The test stimulus was mounted on modified General Radio sweep drive which could oscillate it along an axis in any desired orientation in the subject's frontal parallel plane. The frequency of the achromatic test stimulus oscillations could be varied from 0 to $10 \mathrm{~Hz}$ (fixed amplitude) and monitored electronically.

The subjects were assigned to three groups; Groups 1 and 2 contained 5 subjects each and served as the experimental groups, and Group 3. serving as a control group. contained the other 10 subjects. The subjects in each group were tested individually in a single experimental session. Group 1 viewed the adapting stimuli in orthogonal $45^{\circ}$ orientations, with red gratings at $45^{\circ} \mathrm{cw}$ and green gratings at $45^{\circ} \mathrm{ccw}$ tilt; these contingencies were reversed for Group 2, red gratings being viewed at $45^{\circ} \mathrm{ccw}$ and green gratings at $45^{\circ} \mathrm{cw}$. The procedure for the experimental groups was as follows: Subsequent to viewing the chromatic adapting stimuli, the presence 
of orientation-specitic color aftereffects was determined by presenting the stationary achromatic test pattern with the orthogonal gratings in $45^{\circ}$ orientations. Subjects reporting the presence of color on the stationary contours were then asked to report on the appearance, or on any change in the appearance, of the color as the test stimulus was made to oscillate (oscillation was always along an axis $45^{\circ} \mathrm{cw}$ or ccw). Frequency of the oscillation was increased gradually until the subject reported absence of color on the contours that were orthogonal to the direction of the oscillation. The order of stimulus movement along the axis tilted $\mathrm{cw}$ or ccw was counterbalanced. Group 3 was not exposed to chromatic stimuli; they viewed the stationary achromatic test gratings in $45^{\circ}$ orientations and were asked to report on the presence of any color. and subsequently viewed these contours in motion (similar to Groups 1 and 2), reporting on the emergence of any color.

\section{RESULTS AND DISCUSSION}

All the experimental subjects (Groups 1 and 2) reported the presence of appropriate hues when, following exposure, the black-white test stimulus was presented tilted $45^{\circ}$. Test gratings that were tilted $45^{\circ}$ cw appeared green to subjects in Group 1 and pink to those in Group 2. Oscillation of the stimulus in a direction orthogonal to $45^{\circ} \mathrm{cw}$, that is, oscillating the tilted gratings along an axis tilted $45^{\circ} \mathrm{ccw}$, resulted in enhancement of the green aftereffect for Group 1 (mean $1.67 \mathrm{~Hz}$ ), whereas subjects in Group 2 reported, without exception, that the pink aftereffect had either attenuated substantially or disappeared at the onset of oscillation. Gratings tilted $45^{\circ} \mathrm{ccw}$ appeared pink to Group 1 and green to Group 2. Oscillation of the stimulus orthogonal to $45^{\circ} \mathrm{ccw}$ resulted in the bleaching of the pink aftereffect for Group 1, while resulting in intensitification of the green aftereffect for Group 2 (mean $1.79 \mathrm{~Hz}$ ). Both groups reported the desaturation of pink aftereffects as soon as the stimulus was moved, so no "movement threshold" for attenuation of pink could be determined.

An additional observation is worthy of mention. When the gratings were in an orientation that elicited pink aftereffects, and these were bleached by stimulus movement, cessation of the oscillations resulted in highly transient red rebound; that is, cessation of stimulus movements was followed immediately by momentary intensification of pink well above premovement levels and then return to normal levels. When the achromatic gratings induced green aftereffects, cessation of oscillations resulted in momentary bleaching of green and subsequent rebound to normal levels. Postmovement momentary intensification for pink was reported by $90 \%$ of the subjects, whereas all subjects reported the post movement transient bleaching of green. It is interesting to note that this pattern of postmovement appearance of the McCollough effect resembles disinhibition and inhibition observed in single-unit work.
The data from Group 3 are more complex. Four of the subjects reported no color either on the stationary or moving iest pattern. Three subjects reported the existence of very weak blue-yellow hues on the stationary orthogonal test gratings, with no apparent preference for grating orientation. Upon oscillation of the test stimulus, all three reported enhancement of yellow on the gratings orthogonal to the direction of oscillation, with blue (on the orthogonal gratings) remaining unchanged. Two other subjects reported the emergence of pink immediately upon cessation of movement on gratings oriented in the direction of oscillation (these subjects had reported no color on the stationary stimulus prior to oscillation). Finally, green was reported by one subject during stimulus movement on the grating orthogonal to the direction of movement. The colors reported by these subjects appeared to be transient and quite weak as compared to the chromatic aftereffects subsequently generated.

The results of Groups 1 and 2 clearly show that enhancement and attenuation of the saturation of the orientation-specific color aftereffects by oscillations of the black-white test stimulus is a stable phenomenon, and further that it is color specific. If a grating in a particular orientation elicits green aftereffects, then oscillating that grating in a direction orthogonal to its orientation results in enhancement of that aftereffect; if the same grating in the same orientation elicits pink aftereffects, then the same oscillation results in attenuation of that aftereffect.

The findings from Group 3 cannot be so readily categorized. The fact that four of the subjects reported no color either on the stationary or moving achromatic test pattern, suggests that the oscillation of the gratings, by itself, cannot be solely responsible for the attenuation or enhancement of the chromatic aftereffects reported by the experimental subjects. However, the emergence of color reported by some subjects without prior exposure to chromatic gratings was unexpected and cannot be account for at the present. Highly redundent achromatic patterns, such as moiré patterns, do induce color; however, these are seen as radiating colors, not stationary hues confined to a particular orientation as was the case in this experiment. One is also reminded of the Fechner-Benham colors, which are also induced by moving achromatic stimuli, a phenomenon that could provide at least a partial explanation for the experimental observations. If one could assume that the oscillating achromatic gratings induce color as per Festinger, Allyn, and White's (1971) model for the Fechner-Benham colors, then the most likely color to be induced would be green. Green added to existing green aftereffects would enhance that color and when added to red, desaturate it. This speculation is consistent with the fact that green is enhanced maximally when the achromatic gratings oscillate at 
about $1.8 \mathrm{~Hz}$. At this frequency, a given point on the retina viewing the gratings is stimulated with a light source modulating in a square-wave pattern at about $6 \mathrm{~Hz}$. This is the same frequency at which Festinger et al. (1971) reported obtaining their best green.

While the attenuation of pink aftereffects upon oscillation of the achromatic gratings is consistent with the hypothesis advanced in terms of the Fechner-Benham colors, its occurrence at very slow rates of movement is more difficult to explain. If pink aftereffects were desaturated due to the addition of green, then maximum desaturation should have occurred at the frequency where green appeared strongest, namely at $1.8 \mathrm{~Hz}$. However, as stated above, the fading of pink was almost immediate upon onset of oscillation at low frequencies. Postmovement transient changes in the appearance of the aftereffect could be ascribed to temporary changes in color threshold due to chromatic adaptation generated by the addition of Fechner-Benham green. During oscillation, the intensification of green could produce a temporary shift in the threshold for green such that upon cessation of oscillation the normal green aftereffect would appear less intense. Conversely, desaturation of pink during oscillation could have increased sensitivity to color so that the normal pink aftereffect would appear temporarily to be more intense upon cessation of oscillation. One major shortcoming of this explanation is that the postmovement transient changes are independent of the duration of oscillation. For instance, prolonged oscillation $(1 \mathrm{~min})$ does not reduce the saturation of the concurrent green, which should occur if chromatic adaptation to the more intense color is taking place. Nor is there any change in the duration of the postmovement transient bleaching following long $(1 \mathrm{~min})$ and short $(34 \mathrm{sec})$ periods of stimulus oscillation. The momentary postoscillation intensification of the pink aftereffect also appears to be independent of such temporal factors. Thus, except for the enhancement of green, the relatively short latencies and independence from temporal factors suggest that these phenomena are not likely to be the product solely of adaptation-type processes. Inhibitory and excitatory interactions, in addition to the Fechner-Benham color hypothesis, appear to be necessary to account for the data. At the moment, the mechanisms underlying these phenomena remain unclear.

\section{REFERENCES}

Favreau, O. E., Emerson, V. F., \& Corballis, M. C. Motion perception: A color-contingent aftereffect. Science, 1972, 176. 78-79.

Festinger, L., Allyn. M. R.. \& White, C. W. The perception of color with achromatic stimulation. Vision Research, 1971, 11, 591-612.

HePleR, N. Color-a motion contingent aftereffect. Science, 1968 , 162. $376 \cdot 377$.

Leppman, P. K. Spatial frequency dependent chromatic aftereffects. Nature, 1973, 242, 411-412.

Mayhew, J. E. W., \& Anstis, S. M. Movement aftereffects contingent on color. intensity and pattern. Perception \& Psychophysics, 1972, 12, 77-84.

McCollough, C. Color adaptations of edge-detectors in the human visual system. Science, 1965, 149, 1115-1116.

Riggs, L. A. Curvature as a feature of pattern vision. Science, 1973, 181, 1070-1072.

Rosson, J. G. Spatial and temporal contrast sensitivity functions of the visual system. Journal of the Optical Society of America, 1966, 56. 1141-1142.

White, K. D., \& Riggs, L. A. Angle contingent color aftereffects. Vision Research, 1974, 14, 1147-1154.

(Received for publication October 4, 1974; revision accepted May 23, 1975.) 